\title{
Clinical characteristics and risk factors for mortality in cancer patients with COVID-19
}

Junnan Liang ${ }^{1, *}$, Guannan $\mathrm{Jin}^{2, *}$, Tongtong $\mathrm{Liu}^{3, *}$, Jingyuan Wen ${ }^{1}$, Ganxun $\mathrm{Li}^{1}$, Lin Chen ${ }^{1,3}$, Wei Wang ${ }^{1}$, Yuwei Wang ${ }^{1}$, Wei Liao ${ }^{1}$, Jia Song ${ }^{1}$, Zeyang Ding $(\bowtie)^{1,3}$, Xiao-ping Chen $(\bowtie)^{1,3}$, Bixiang Zhang $(\bowtie)^{1,3}$

${ }^{1}$ Hepatic Surgery Center, Liver Cancer Institute, and Hubei Key Laboratory of HPB Diseases, Tongji Hospital, Tongji Medical College, Huazhong University of Science and Technology, Wuhan 430030, China; ${ }^{2}$ Department of Internal Medicine, Union Hospital, Tongji Medical College, Huazhong University of Science and Technology, Wuhan 430030, China; ${ }^{3}$ Tongji Multidisciplinary Team for Treating COVID-19 (TTTC), Tongji Hospital, Tongji Medical College, Huazhong University of Science and Technology, Wuhan 430030, China

(C) Higher Education Press 2021

Abstract Patients with cancer are at increased risk of severe infections. From a cohort including 3060 patients with confirmed COVID-19, 109 (3.4\%) cancer patients were included in this study. Among them, 23 (21.1\%) patients died in the hospital. Cancer patients, especially those with hematological malignancies $(41.6 \%)$, urinary carcinoma (35.7\%), malignancies of the digestive system (33.3\%), gynecological malignancies (20\%), and lung cancer $(\mathbf{1 4 . 3 \%})$, had a much higher mortality than patients without cancer. A total of $19(17.4 \%)$ cancer patients were infected in the hospital. The clinical characteristics of deceased cancer patients were compared with those of recovered cancer patients. Multivariate Cox regression analysis indicated that a Nutritional Risk Screening (NRS2002) score $\geqslant 3$ (adjusted hazard ratio (HR) 11.00; 95\% confidence interval (CI) 4.60-26.32; $P<0.001$ ), high-risk type (adjusted HR 18.81; 95\% CI 4.21-83.93; $P<0.001$ ), tumor stage IV (adjusted HR 4.26; 95\% CI 2.34-7.75; $P<0.001)$, and recent adjuvant therapy (<1 month) (adjusted HR 3.16; 95\% CI 1.75-5.70; $P<0.01)$ were independent risk factors for in-hospital death after adjusting for age, comorbidities, D-dimer, and lymphocyte count. In conclusion, cancer patients showed a higher risk of COVID-19 infection with a poorer prognosis than patients without cancer. Cancer patients with high-risk tumor, NRS2002 score $\geqslant 3$, advanced tumor stage, and recent adjuvant therapy $(<1$ month) may have high risk of mortality.

Keywords cancer; COVID-19; SARS-CoV-2; risk factor; mortality

\section{Introduction}

Since December 2019, coronavirus disease 2019 (COVID19) has resulted in considerable morbidity and mortality around the world $[1,2]$.

Cancer is among the most prevalent diseases with more than 18 million new cases per year globally. Cancer patients are vulnerable to infectious diseases due to compromised host defenses and sequelae of antineoplastic treatment [3-5]. Therefore, the effects of COVID-19 on cancer patients have received wide attention. A previous

Received August 27, 2020; accepted December 29, 2020

Correspondence: Xiao-ping Chen, chenxpchenxp@163.com; Bixiang Zhang, bixiangzhang@hust.edu.cn; Zeyang Ding, dingzeyang@hust.edu.cn

\footnotetext{
${ }^{*}$ These authors contributed equally to this work.
}

report found that cancer patients had a higher risk for severe events and mortality compared with COVID-19 patients without cancer [6]. In another report, Zhou et al. confirmed that patients who had received antitumor treatment in the preceding 14 days or presented with patchy consolidation on the first lung computed tomography (CT) on admission had an elevated risk of developing severe COVID-19-related events [7]. Considering the small sample size and limited clinical information, previous reports were not amenable to more insightful analyses, such as assessing the risk of in-hospital death, the clinical characteristics of deceased patients, and the effect on different types of cancer and even curative cancer treatments in the setting of SARS-CoV-2 infection.

As the epidemic continues to spread around the world, the clinical characteristics of deceased cancer patients should be investigated, and the risk factors associated with in-hospital death of cancer patients with COVID-19 should 
be identified. In this study, we conducted a retrospective cohort study of 109 COVID-19 patients with cancer and definite outcome (discharged or died) from Tongji Hospital in Wuhan, China. We first compared the demographic, clinical, laboratory, and radiological features of cancer patients with different clinical outcomes to conduct a comprehensive evaluation of clinical characteristics of deceased cancer patients and explore the risk factors associated with in-hospital death among cancer patients with COVID-19 through multivariate Cox regression analysis.

\section{Methods}

\section{Study population}

We included 109 consecutive patients with confirmed COVID-19 pneumonia admitted to Tongji Hospital in Wuhan, China from January 18, 2020 to March 20, 2020 with definite outcome (discharged or died) and long-term follow-up (as of April 15, 2020, the last patient had been discharged). Among the 109 patients, 14 patients have been described in a previous study [7]. During the outbreak of COVID-19, Tongji Hospital was designated as a hospital for the treatment of severe COVID-19 patients by the Chinese government. All patients enrolled in this study were diagnosed as having COVID-19 pneumonia according to the interim guidance of the World Health Organization. The study protocol was approved by the Institutional Review Board of Tongji Hospital, Tongji Medical College, Huazhong University of Science and Technology (Wuhan, China) (Approval No. TJIRB20200408).

\section{Data collection}

The epidemiological, clinical, laboratory, and radiological characteristics and treatment and outcome data of participants collected during hospitalization were obtained from electronic medical records using data collection forms. The demographics, medical history, exposure history, underlying chronic diseases, symptoms and signs, laboratory findings, chest CT scans, and treatments (including antiviral therapy, antibiotics, corticosteroid therapy, and oxygen support) during hospital admission were also collected from the electronic medical records. The data were reviewed by a trained team of surgeons. All clinical data were collected up to April 15, 2020, the final date of follow-up. Patients were categorized according to the presence of cancer. Acute respiratory distress syndrome (ARDS) was diagnosed according to the Berlin definition. Acute kidney injury was identified according to the guidelines of Kidney Disease: Improving Global Outcomes. The details of clinical indicators and definitions of complications are available in Table S1.

To confirm COVID-19, an RNA isolation kit (Biogerm, Shanghai, China) was used to extract SARS-CoV-2 RNA from throat-swab specimens of patients. A SARS-CoV-2 nucleic acid detection kit was used to detect SARS-CoV-2 RNA via real-time reverse transcriptase-polymerase chain reaction, according to the manufacturer's instructions.

\section{Statistical analysis}

SPSS version 26.0 (IBM Corp., USA) and PRISM version 6.0. (GraphPad 153 Software Inc., USA) were used for statistical analysis. Descriptive analyses of categorical parameters were expressed as frequency rates and percentages, whereas continuous parameters were expressed as mean, median, and interquartile range (IQR) values. Continuous data were compared using one-way ANOVA or the Kruskal-Wallis test. The $\chi^{2}$ test or Fisher exact test was performed for categorical data. Univariate and multivariate Cox regression models were used to determine the hazard ratios (HRs) and 95\% confidence intervals (CIs) of the risk factors of death in cancer patients. In full multivariate models, we assessed the interactions of cancer indicators with age, comorbidities, D-dimer, and lymphocyte count on the basis of previous findings and clinical characteristics of cancer patients, including TNM stage, NRS2002 score, recent adjuvant therapy ( $<1$ month), and cancer type [8-11]. The comorbidities included chronic obstructive pulmonary disease, hypertension, diabetes, and cardiovascular disease. The Kaplan-Meier method with a log-rank test was used to plot survival curves. For all analyses, a two-sided $P$ value of less than 0.05 was considered to indicate statistical significance.

\section{Results}

\section{Demographic and clinical characteristics}

Among 3060 patients who were hospitalized in Tongji Hospital between January 18, 2020 and March 20, 2020, a total of $109(3.3 \%)$ cancer patients with confirmed COVID-19 and definite outcome were identified and enrolled in this study. Of these patients, $252(8.2 \%)$ had died, and 2808 patients fully recovered and were discharged. Among the 109 cancer patients, 23 (21.1\%) died of COVID-19, and 86 (78.9\%) fully recovered and were discharged. All patients were local residents of the three main districts of Wuhan, China. The demographic and clinical characteristics of patients are presented in Table 1. The median age of deceased patients was 68.00 years (IQR 53.50, 76.50), which was higher than that of recovered patients at $64.00(56.00,70.00)$. A total of 13 $(56.5 \%)$ deceased patients and $42(48.8 \%)$ recovered 
Table 1 Clinical characteristics of cancer patients with COVID-19

\begin{tabular}{|c|c|c|c|c|}
\hline Variables & $\begin{array}{l}\text { All patients } \\
(n=109)\end{array}$ & $\begin{array}{l}\text { Recovered } \\
(n=86)\end{array}$ & $\begin{array}{r}\text { Deceased } \\
(n=23)\end{array}$ & $P$ value \\
\hline Age (year, median (IQR)) & $65.00(56.00,71.00)$ & $64.00(56.00,70.00)$ & $68.00(53.50,76.50)$ & 0.33 \\
\hline Age $\geqslant 65$ years & $55(50.5)$ & $42(48.8)$ & $13(56.5)$ & 0.675 \\
\hline \multicolumn{5}{|l|}{ Sex } \\
\hline Female & $52(47.7)$ & $43(50.0)$ & $9(39.1)$ & 0.489 \\
\hline Male & $57(52.3)$ & $43(50.0)$ & $14(60.9)$ & 0.489 \\
\hline \multicolumn{5}{|l|}{ Source of infection } \\
\hline Nosocomial transmission* & $19(17.5)$ & $11(12.8)$ & $8(34.8)$ & 0.014 \\
\hline Community infection $* *$ & $90(82.5)$ & $75(87.2)$ & $15(65.2)$ & 0.014 \\
\hline During out-of-hospital treatment & $6(5.5)$ & $2(2.3)$ & $4(17.4)$ & $<0.001$ \\
\hline Follow-up period & $84(77.0)$ & $73(84.9)$ & $11(47.8)$ & $<0.001$ \\
\hline \multicolumn{5}{|l|}{ Signs and symptoms at admission } \\
\hline Fever & $86(78.9)$ & $68(79.1)$ & $18(78.3)$ & 1 \\
\hline Cough & $75(68.8)$ & $59(68.6)$ & $16(69.6)$ & 1 \\
\hline Expectoration & $49(45.0)$ & $37(43.0)$ & $12(52.2)$ & 0.584 \\
\hline Shortness of breath & $51(46.8)$ & $39(45.3)$ & $12(52.2)$ & 0.728 \\
\hline Pharyngalgia & $5(4.6)$ & $4(4.7)$ & $1(4.3)$ & 1 \\
\hline Rhinorrhoea & $3(2.8)$ & $2(2.3)$ & $1(4.3)$ & 1 \\
\hline Fatigue & $25(22.9)$ & $18(20.9)$ & $7(30.4)$ & 0.494 \\
\hline Chest pain & $8(7.3)$ & $7(8.1)$ & $1(4.3)$ & 0.866 \\
\hline Diarrhea & $26(23.9)$ & $20(23.3)$ & $6(26.1)$ & 0.994 \\
\hline Abdominal pain & $5(4.6)$ & $3(3.5)$ & $2(8.7)$ & 0.618 \\
\hline Anorexia & $26(23.9)$ & $21(24.4)$ & $5(21.7)$ & 1 \\
\hline Nausea or vomiting & $10(9.2)$ & $7(8.1)$ & $3(13.0)$ & 0.751 \\
\hline Myalgia & $14(12.8)$ & $11(12.8)$ & $3(13.0)$ & 1 \\
\hline Headache & $9(8.3)$ & $7(8.1)$ & $2(8.7)$ & 1 \\
\hline Dizziness & $8(7.3)$ & $7(8.1)$ & $1(4.3)$ & 0.866 \\
\hline Respiratory rate, breath per minute & $20.00(20.00,22.00)$ & $20.00(20.00,22.00)$ & $20.00(20.00,22.50)$ & 0.661 \\
\hline Pulse, beat per minute & $86.00(76.00,98.00)$ & $85.00(76.00,97.75)$ & $89.00(78.00,105.50)$ & 0.296 \\
\hline Median arterial pressure, $\mathrm{mmHg}$ & $96.33(90.67,103.33)$ & $96.83(92.17,103.58)$ & $93.00(83.83,101.00)$ & 0.127 \\
\hline Percutaneous oxygen saturation, $\%$ & $96.00(92.00,99.00)$ & $96.50(94.25,99.00)$ & $89.00(75.00,97.00)$ & 0.005 \\
\hline \multicolumn{5}{|l|}{ Chronic comorbidities } \\
\hline Hypertension & $38(34.9)$ & $33(38.4)$ & $5(21.7)$ & 0.215 \\
\hline Diabetes & $18(16.5)$ & $15(17.4)$ & $3(13.0)$ & 0.85 \\
\hline Cardiovascular disease & $10(9.2)$ & $9(10.5)$ & $1(4.3)$ & 0.62 \\
\hline Cerebrovascular disease & $4(3.7)$ & $4(4.7)$ & $0(0.0)$ & 0.668 \\
\hline Chronic pulmonary disease & $19(17.4)$ & $17(19.8)$ & $2(8.7)$ & 0.35 \\
\hline Chronic kidney disease & $3(2.8)$ & $3(3.5)$ & $0(0.0)$ & 0.849 \\
\hline Chronic liver disease & $10(9.2)$ & $6(7.0)$ & $4(17.4)$ & 0.258 \\
\hline \multicolumn{5}{|l|}{ Clinical type on admission } \\
\hline Severe pneumonia & $60(55.0)$ & $44(51.2)$ & $16(69.6)$ & 0.18 \\
\hline Days from illness onset to outcome & $41.28(29.00,54.50)$ & $45.50(33.00,56.26)$ & $25.52(15.00,33.00)$ & $<0.001$ \\
\hline Days from admission to outcome & $23.72(13.24,36.63)$ & $26.67(17.51,41.12)$ & $7.62(4.44,17.25)$ & $<0.001$ \\
\hline
\end{tabular}

Data are presented as number/total (percentage) or median (IQR); $P$ values were calculated using the Mann-Whitney $\mathrm{U}$ test, $\chi^{2}$ test, or Fisher's exact test, as appropriate. COVID-19, coronavirus disease 2019. The severity was staged based on the guidelines for the diagnosis and treatment of COVID-19 (trial seventh edition) published by the Chinese National Health Commission on February 4, 2020.

* indicates that the most common department of nosocomial infection was hematology $(7,36.8 \%)$ followed by the departments of oncology $(5,26.3 \%)$, general surgery $(3,15.8 \%)$, urinary surgery $(1,5.3 \%)$, gynecology $(1,5.3 \%)$, thoracic surgery $(1,5.3 \%)$, and critical care medicine $(1,5.3 \%)$.

$* *$ indicates that community-infected patients included two patients who were within one month after discharge.

patients were aged 65 years or older. A majority of deceased patients were male $(14,60.9 \%)$ compared with half of recovered patients $(43,50.0 \%)$. Fever and cough were the most common symptoms at disease onset in both 
deceased patients $(18,78.3 \% ; 16,69.6 \%)$ and recovered patients $(68,79.1 \% ; 59,68.6 \%)$. The proportions of other prevalent symptoms and comorbidities in the two groups were comparable. The percutaneous oxygen saturation on admission in patients who succumbed (89.00 (75.00, 97.00)) was substantially lower than that in patients who recovered $(96.50(94.25,99.00))(P=0.005)$. Moreover, a total of $19(17.4 \%)$ cancer patients were infected in the hospital, and the proportion of nosocomial infection among patients who succumbed $(8,34.8 \%)$ was significantly higher than that among patients who recovered (11, 12.8\%) $(P=0.014)$. The most common department for nosocomial infection was hematology $(7,36.8 \%)$, followed by the departments of oncology $(5,26.3 \%)$, general surgery $(3,15.8 \%)$, urinary surgery $(1,5.3 \%)$, gynecology $(1,5.3 \%)$, thoracic surgery $(1,5.3 \%)$, and critical care medicine $(1,5.3 \%)$. The proportion of patients who were infected during out-of-hospital treatment was much higher among the deceased patients $(4,17.4 \%)$ than among the recovered patients $(2,2.3 \%)(P<0.001)$. The median time from illness onset to death was $25.52(15.00,33.00)$ days, and the median time from illness onset to discharge among recovered patients was $45.50(33.00,56.26)$ days $(P<0.001)$. The median time from admission to death was $7.62(4.44,17.25)$ days, and the median time from admission to discharge was $26.67(17.51,41.12)$ days $(P<0.001)$.

\section{Oncological characteristics}

As shown in Table 2, among the 109 cancer patients, malignancies of the digestive system $(24,22 \%)$, head and neck cancer $(21,19.3 \%)$, lung cancer $(14,12.8 \%)$, and urinary carcinoma $(14,12.8 \%)$ were the most frequent cancer type. Among the deceased patients, malignancies of the digestive system were the most frequent type of cancer $(8,34.8 \%)$, followed by hematological malignancies $(5$, $21.7 \%)$ and urinary carcinoma $(5,21.7 \%)$. Compared with patients who recovered, hematological malignancies and malignancies of the digestive system were more frequent among deceased patients $(5,21.7 \%$ vs. $7,8.1 \% ; P=0.064$; $8,34.8 \%$ vs. $16.18 .6 \% ; P=0.096)$. As shown in Fig. S1, patients with hematological malignancies had the highest death rate $(41.6 \%)$, followed by urinary carcinoma (35.7\%), malignancies of the digestive system (33.3\%), gynecological malignancies (20\%), and lung cancer $(14.3 \%)$. We classified these tumors as high-risk type because their death rate is much higher than that of the general population. Among the deceased patients, two (8.7\%) had an Eastern Cooperative Oncology Group (ECOG) performance status score $>2$, and $20(86.9 \%)$ had a Nutritional Risk Screening 2002 (NRS2002) score $\geqslant 3$. The odds of patients who had an ECOG performance status score $>2$ or NRS2002 score $\geqslant 3$ were significantly higher among deceased patients than among recovered patients $(1,1.2 \% ; 19,22.1 \%)(P=0.049$; $P<0.001)$. A total of $12(52.1 \%)$ deceased patients were diagnosed with metastatic cancer (stage IV), which was a significantly higher proportion than that of patients who recovered $(11,12.3 \%)(P<0.001)$. Among the 109 COVID-19 patients with cancer in our study, 17 (15.6\%) had received chemo-/radiotherapy, 4 (3.7\%) underwent surgery ( 3 patients during the current hospitalization), and 3 (2.7\%) had targeted/immunotherapy within 1 month before the onset of COVID-19 symptoms. The targeted/ immunotherapy included PD1 inhibitors for the treatment of ovarian cancer, chimeric antigen receptor $\mathrm{T}$ cell immunotherapy for the treatment of multiple myeloma, and vascular endothelial growth factor inhibitor (bevacizumab injection) for the treatment of lung adenocarcinoma. All patients discontinued anticancer treatment after SARS-CoV-2 infection was confirmed. Likewise, the proportion of patients who received adjuvant therapy, recent adjuvant therapy ( $<1$ month), chemo-/radiotherapy, recent chemo-/radiotherapy $(<1$ month $)$, targeted/ immunotherapy, and recent targeted/immunotherapy $(<1$ month) was significantly higher among deceased patients $(19,82.6 \% ; 11,47.8 \% ; 16,69.5 \%, 10,43.4 \% ; 5,21.7 \%$; and $2,8.7 \%$, respectively) than among recovered patients $(40,46.5 \% ; 9,10.4 \% ; 38,44.2 \% ; 8,9.3 \% ; 4,4.7 \%$; and 1 , $1.2 \%$, respectively) $(P=0.002, P<0.001, P=0.031$, $P<0.001, P=0.008$, and $P=0.049$, respectively).

\section{Laboratory and radiological findings, treatment, and complications}

The laboratory and radiological findings on admission are summarized in Table S2. Compared with recovered patients, deceased patients had many differences in laboratory indices, such as lymphocyte count, platelet count, and direct bilirubin. As shown in Table 3, more deceased patients received glucocorticoid therapy (23, $100.0 \%)$ and intravenous immunoglobin $(12,52.2 \%)$ than recovered patients $(31,36.0 \% ; 23,26.7 \%)$. Significantly more deceased patients were subjected to mechanical ventilation (noninvasive: $14,60.9 \%$ vs. $8,9.3 \%$, $P<0.001$; invasive: $6,26.1 \%$ vs. $2,2.3 \%, P<0.001$ ) than recovered patients. Among the deceased patients, common complications included sepsis $(23,100.0 \%)$, ARDS $(20,87.0 \%)$, acute cardiac injury $(17,73.9 \%)$, and heart failure $(17,73.9 \%)$. These complications were significantly more frequent in deceased patients than in recovered patients $(P<0.001$ in all cases), suggesting their potential association with the clinical outcome. Of the 23 deceased patients, 20 (86.9\%) died of COVID-19, and three (13.1\%) died of cancer. As shown in Table S3, the causes of death included respiratory failure $(10,43.5 \%)$, septic shock $(5,21.7 \%)$, multiple organ failure $(3,13.0 \%)$, 
Table 2 Oncological characteristics of cancer patients with COVID-19

\begin{tabular}{|c|c|c|c|c|}
\hline Variables & $\begin{array}{l}\text { All patients } \\
(n=109)\end{array}$ & $\begin{array}{c}\text { Recovered } \\
\quad(n=86)\end{array}$ & $\begin{array}{r}\text { Deceased } \\
(n=23)\end{array}$ & $P$ value \\
\hline \multicolumn{5}{|l|}{ Tumor type } \\
\hline Lung cancer & $14(12.8)$ & $12(13.9)$ & $2(8.2)$ & 0.503 \\
\hline Malignancy of the digestive system & $24(22.0)$ & $16(18.6)$ & $8(34.8)$ & 0.096 \\
\hline Gastric carcinoma & $8(7.3)$ & $5(5.8)$ & $3(13.0)$ & 0.237 \\
\hline Colorectal cancer & $5(4.6)$ & $4(4.7)$ & $1(4.3)$ & 0.949 \\
\hline Esophagus cancer & $4(3.7)$ & $2(2.3)$ & $2(8.7)$ & 0.149 \\
\hline Rectal cancer & $2(1.8)$ & $2(2.3)$ & 0 & 0.460 \\
\hline Liver cancer & $5(4.6)$ & $3(3.5)$ & $2(8.7)$ & 0.289 \\
\hline Head and neck cancer & $21(19.3)$ & $21(24.4)$ & 0 & 0.008 \\
\hline Laryngeal carcinoma & $1(0.9)$ & $1(1.2)$ & 0 & 0.603 \\
\hline Oral cancer & $1(0.9)$ & $1(1.2)$ & 0 & 0.603 \\
\hline Nasopharyngeal carcinoma & $3(2.7)$ & $3(3.5)$ & 0 & 0.363 \\
\hline Thyroid cancer & $16(14.7)$ & $16(18.6)$ & 0 & 0.025 \\
\hline Hematological malignancies & $12(11.0)$ & $7(8.1)$ & $5(21.7)$ & 0.064 \\
\hline Leukemia & $7(6.4)$ & $4(4.7)$ & $3(13.0)$ & 0.144 \\
\hline Lymphoma & $4(3.7)$ & $3(3.5)$ & $1(4.3)$ & 0.845 \\
\hline Multiple myeloma & $1(0.9)$ & 0 & $1(4.3)$ & 0.052 \\
\hline Urinary carcinoma & $14(12.8)$ & $9(10.5)$ & $5(21.7)$ & 0.151 \\
\hline Bladder cancer & $8(7.3)$ & $4(4.7)$ & $4(17.4)$ & 0.037 \\
\hline Prostate cancer & $4(3.7)$ & $3(3.5)$ & $1(4.3)$ & 0.845 \\
\hline Renal carcinoma & $2(1.8)$ & $2(2.3)$ & 0 & 0.460 \\
\hline Breast cancer & $11(10.1)$ & $11(12.8)$ & 0 & 0.070 \\
\hline Gynecological malignancies & $10(9.2)$ & $8(9.3)$ & $2(8.7)$ & 0.928 \\
\hline Uterine malignancy & $4(3.7)$ & $3(3.5)$ & $1(4.3)$ & 0.845 \\
\hline Cervical cancer & $5(4.6)$ & $4(4.7)$ & $1(4.3)$ & 0.949 \\
\hline Ovarian cancer & $1(0.9)$ & $1(1.2)$ & 0 & 0.603 \\
\hline Others & $3(2.7)$ & $2(2.3)$ & $1(4.3)$ & 0.312 \\
\hline Spinal tumor & $1(0.9)$ & $1(1.2)$ & 0 & 0.598 \\
\hline Bronchial tumor & $1(0.9)$ & $1(1.2)$ & 0 & 0.603 \\
\hline Liposarcoma & $1(0.9)$ & 0 & $1(4.3)$ & 0.052 \\
\hline \multicolumn{5}{|l|}{ Condition grading } \\
\hline Karnofsky performance score $(\geqslant 70)$ & $8(7.3)$ & $5(5.8)$ & $3(13.0)$ & 0.237 \\
\hline ECOG performance status score $(>2)$ & $3(2.7)$ & $1(1.2)$ & $2(8.7)$ & 0.049 \\
\hline NRS2002 score $(\geqslant 3)$ & $39(35.7)$ & $19(22.1)$ & $20(86.9)$ & $<0.001$ \\
\hline \multicolumn{5}{|l|}{ Tumor stage } \\
\hline Stage I/II/III & $86(78.8)$ & $75(87.2)$ & $11(47.8)$ & $<0.001$ \\
\hline Stage IV & $23(21.2)$ & $11(12.3)$ & $12(52.1)$ & $<0.001$ \\
\hline \multicolumn{5}{|l|}{ History of prior treatment } \\
\hline Operation & $65(59.6)$ & $55(64.0)$ & $10(43.4)$ & 0.075 \\
\hline Operation ( $<1$ month) & $4(3.7)$ & $4(4.7)$ & 0 & 0.291 \\
\hline Adjuvant therapy & $59(54.1)$ & $40(46.5)$ & $19(82.6)$ & 0.002 \\
\hline Recent adjuvant therapy & $20(18.3)$ & $8(9.3)$ & $12(52.1)$ & $<0.001$ \\
\hline Chemo-/radiotherapy & $54(49.5)$ & $38(44.2)$ & $16(69.5)$ & 0.031 \\
\hline Recent chemo-/radiotherapy ( $<1$ month) & $17(15.6)$ & $7(8.1)$ & $10(43.4)$ & $<0.001$ \\
\hline Targeted/immunotherapy & $9(8.2)$ & $4(4.7)$ & $5(21.7)$ & 0.008 \\
\hline Recent targeted/immunotherapy ( $<1$ month) & $3(2.7)$ & $1(1.2)$ & $2(8.7)$ & 0.049 \\
\hline
\end{tabular}

Data are presented as number/total (percentage) or median (IQR); $P$ values were calculated using the Mann-Whitney U test, $\chi^{2}$ test, or Fisher's exact test, as appropriate. COVID-19, coronavirus disease 2019; ECOG, Eastern Cooperative Oncology Group; NRS2002, Nutritional Risk Screening 2002; adjuvant therapy, chemo-/radiotherapy plus targeted/immunotherapy. 
Table 3 Treatment and complications in cancer patients with COVID-19

\begin{tabular}{|c|c|c|c|c|}
\hline Variables & $\begin{array}{l}\text { All patients } \\
(n=109)\end{array}$ & $\begin{array}{l}\text { Recovered } \\
(n=86)\end{array}$ & $\begin{array}{r}\text { Deceased } \\
(n=23)\end{array}$ & $P$ value \\
\hline \multicolumn{5}{|l|}{ Medicine therapy } \\
\hline Antibiotic treatment & $86(78.9)$ & $63(73.3)$ & $23(100.0)$ & 0.012 \\
\hline Antiviral treatment & $93(85.3)$ & $73(84.9)$ & $20(87.0)$ & 0.803 \\
\hline Arbidol & $88(80.7)$ & $69(80.2)$ & $19(82.6)$ & 0.797 \\
\hline Lopinavir/ritonavir & $28(25.7)$ & $22(25.9)$ & $6(26.1)$ & 0.964 \\
\hline Oseltamivir & $18(16.5)$ & $15(17.4)$ & $3(13.0)$ & 0.613 \\
\hline Ganciclovir & $17(15.6)$ & $11(12.8)$ & $6(26.1)$ & 0.118 \\
\hline Hydroxychloroquine & $21(19.2)$ & $18(20.9)$ & $3(13.0)$ & 0.394 \\
\hline Interferon & $14(12.8)$ & $11(12.8)$ & $2(13.0)$ & 0.974 \\
\hline Ribavirin & $11(10.1)$ & $10(11.6)$ & $1(4.3)$ & 0.303 \\
\hline Combination ( $>1$ antiviral drugs) & $94(86.2)$ & $74(86.0)$ & $20(87.0)$ & 0.909 \\
\hline Traditional Chinese medicine treatment & $93(85.3)$ & $78(90.7)$ & $15(65.2)$ & 0.006 \\
\hline Glucocorticoid therapy & $54(49.5)$ & $31(36.0)$ & $23(100.0)$ & $<0.001$ \\
\hline NSAIDs & $24(22.0)$ & $17(19.8)$ & $7(30.4)$ & 0.416 \\
\hline Intravenous immunoglobin & $35(32.1)$ & $23(26.7)$ & $12(52.2)$ & 0.039 \\
\hline \multicolumn{5}{|l|}{ Physiotherapy } \\
\hline Mechanical ventilation & $30(27.5)$ & $10(11.6)$ & $20(87.0)$ & $<0.001$ \\
\hline Noninvasive & $22(20.2)$ & $8(9.3)$ & $14(60.9)$ & $<0.001$ \\
\hline Invasive & $8(7.3)$ & $2(2.3)$ & $6(26.1)$ & 0.001 \\
\hline \multicolumn{5}{|l|}{ Complications } \\
\hline ARDS & $31(28.4)$ & $11(12.8)$ & $20(87.0)$ & $<0.001$ \\
\hline Heart failure & $27(29.3)$ & $10(13.7)$ & $17(73.9)$ & $<0.001$ \\
\hline Liver injury & $12(11.0)$ & $6(7.0)$ & $6(26.1)$ & 0.009 \\
\hline AKI & $10(9.2)$ & $4(4.7)$ & $6(26.1)$ & 0.006 \\
\hline Acute cardiac injury & $32(34.8)$ & $15(20.5)$ & $17(73.9)$ & $<0.001$ \\
\hline Sepsis & $27(24.8)$ & $4(4.7)$ & $23(100.0)$ & $<0.001$ \\
\hline
\end{tabular}

Data are presented as number/total (percentage) or median (IQR); $P$ values were calculated using the Mann-Whitney U test, $\chi^{2}$ test, or Fisher's exact test, as appropriate. COVID-19, coronavirus disease 2019; NSAIDs, nonsteroidal anti-inflammatory drugs; ARDS, acute respiratory distress syndrome; AKI, acute kidney injury.

acute heart failure $(3,13.0 \%)$, acute myocardial infarction $(1,4.3 \%)$, and others $(1,4.3 \%)$. The death records of three patients who died of cancer are shown in Table S4.

\section{Risk factors for death in cancer patients with comorbid COVID-19}

The association of clinical factors with death according to the univariate and multivariate Cox proportional hazards model is summarized in Table 4 and Fig. 1. In univariate analysis, percutaneous oxygen saturation $(\leqslant 93 \%)$, type of malignancy (high-risk type), tumor stage IV, ECOG performance status score $(>2)$, NRS2002 score $(\geqslant 3)$, adjuvant therapy, recent adjuvant therapy $(<1$ month), chemo-/radiotherapy, recent chemo-/radiotherapy $(<1$ month), targeted/immunotherapy, nosocomial infection, platelet count $\left(<125 \times 10^{9} / \mathrm{L}\right)$, D-dimer $(>1 \mu \mathrm{g} / \mathrm{mL})$, aspartate aminotransferase $(>40 \mathrm{U} / \mathrm{L})$, albumin $(<35 \mathrm{~g} / \mathrm{L})$, high-sensitivity cardiac troponin I $(>15.6 \mathrm{pg} / \mathrm{mL}), \mathrm{N}-$ terminal pro-brain natriuretic peptide $(>486 \mathrm{pg} / \mathrm{mL})$, highly sensitive C-reactive protein $(>10 \mathrm{mg} / \mathrm{L})$, procalcitonin $(>0.05 \mathrm{ng} / \mathrm{mL})$, and IL-10 ( $>9.1 \mathrm{pg} / \mathrm{mL})$ were significantly associated with higher risk of death among cancer patients with COVID-19. In multivariate analysis, NRS2002 score $\geqslant 3$ (adjusted HR 11.00; 95\% CI 4.60-26.32; $P<0.001$ ), high-risk type (adjusted HR 18.81; 95\% CI 4.21-83.93; $P<0.001$ ), tumor stage IV (adjusted HR 4.26; 95\% CI 2.34-7.75; $P<0.001)$, and recent adjuvant therapy ( $<1$ month) (adjusted HR 3.16; 95\% CI 1.75-5.70; $P<0.01)$ were associated with increased odds of inhospital death after adjusting for potential confounders, including age, other comorbidities, D-dimer, and lymphocyte count. The adjusted Kaplan-Meier estimates of survival for these prognostic factors are shown in Fig. 2.

Because cancer contributes an independent risk for death, we further analyzed the association of these risk factors with mortality of COVID-19 in cancer patients using competing risk models to further avoid the potential 
Table 4 Univariate Cox regression analysis of risk factors associated with death in cancer patients with COVID-19

\begin{tabular}{|c|c|c|}
\hline \multirow{2}{*}{ Patient characteristics and findings } & \multicolumn{2}{|c|}{ Univariate } \\
\hline & $\overline{\mathrm{HR}}(95 \% \mathrm{CI})$ & $P$ value \\
\hline Age, $>65$ years & $1.528(0.668-3.493)$ & 0.315 \\
\hline Sex, male & $1.399(0.605-3.234)$ & 0.433 \\
\hline \multicolumn{3}{|l|}{ Chronic comorbidities } \\
\hline Chronic pulmonary disease & $0.396(0.093-1.689)$ & 0.211 \\
\hline \multicolumn{3}{|l|}{ Signs and symptoms at admission } \\
\hline Percutaneous oxygen saturation, $\leqslant 93 \%$ & $3.345(1.473-7.599)$ & 0.004 \\
\hline \multicolumn{3}{|l|}{ Tumor type } \\
\hline High-risk type & $11.094(1.495-82.35)$ & 0.019 \\
\hline \multicolumn{3}{|l|}{ Tumor stage } \\
\hline Tumor stage IV & $6.025(2.647-13.713)$ & $<0.001$ \\
\hline \multicolumn{3}{|l|}{ Condition grading } \\
\hline Karnofsky performance score $(\geqslant 70)$ & $0.512(0.152-1.724)$ & 0.28 \\
\hline ECOG performance status score $(>2)$ & $4.95(1.152-53.191)$ & 0.032 \\
\hline NRS2002 score $(\geqslant 3)$ & $14.53(4.31-48.91)$ & $<0.001$ \\
\hline \multicolumn{3}{|l|}{ History of prior treatment } \\
\hline Operation & $0.566(0.248-1.292)$ & 0.176 \\
\hline a. Operation (<1 month) & $1.304(0.174-9.757)$ & 0.796 \\
\hline Adjuvant therapy & $5.137(1.743-15.139)$ & 0.003 \\
\hline Recent adjuvant therapy ( $<1$ month) & $4.194(1.845-9.534)$ & $<0.001$ \\
\hline Chemo-/radiotherapy & $2.589(1.064-6.301)$ & 0.036 \\
\hline b. Recent chemo-/radiotherapy ( $<1$ month) & $4.834(2.116-11.043)$ & $<0.001$ \\
\hline Targeted/immunotherapy & $3.662(1.352-9.914)$ & 0.011 \\
\hline c. Recent targeted/immunotherapy ( $<1$ month) & $3.458(0.803-14.898)$ & 0.096 \\
\hline Combined $(\mathrm{a}+\mathrm{b}$ or $\mathrm{a}+\mathrm{c})$ & $3.786(0.507-28.29)$ & 0.195 \\
\hline \multicolumn{3}{|l|}{ Source of infection } \\
\hline Nosocomial infection & $2.501(1.56-5.923)$ & 0.037 \\
\hline \multicolumn{3}{|l|}{ Chest computed tomography scan features } \\
\hline Bilateral & $1.071(0.134-8.568)$ & 0.948 \\
\hline \multicolumn{3}{|l|}{ Laboratory findings } \\
\hline Lymphocyte count, $<1.1 \times 10^{9} / \mathrm{L}$ & $2.874(0.976-8.468)$ & 0.055 \\
\hline Platelet count, $<125 \times 10^{9} / \mathrm{L}$ & $6.752(2.859-15.951)$ & $<0.001$ \\
\hline Hemoglobin, $<120 \mathrm{~g} / \mathrm{L}$ & $2.006(0.868-4.638)$ & 0.104 \\
\hline D-dimer, $>1 \mu \mathrm{g} / \mathrm{mL}$ & $4.018(1.365-11.828)$ & 0.012 \\
\hline Aspartate aminotransferase, $>40 \mathrm{U} / \mathrm{L}$ & $2.516(1.1-5.751)$ & 0.029 \\
\hline Total bilirubin, $>21.1 \mu \mathrm{mol} / \mathrm{L}$ & $5.000(1.96-12.5)$ & 0.001 \\
\hline Direct bilirubin, $>8 \mu \mathrm{mol} / \mathrm{L}$ & $2.85(1.204-6.666)$ & 0.018 \\
\hline Albumin, $<35 \mathrm{~g} / \mathrm{L}$ & $3.823(1.411-10.25)$ & 0.008 \\
\hline Lactose dehydrogenase, $>214 \mathrm{U} / \mathrm{L}$ & $2.394(0.879-6.521)$ & 0.088 \\
\hline High-sensitivity cardiac troponin $\mathrm{I},>15.6 \mathrm{pg} / \mathrm{mL}$ & $7.081(2.782-18.026)$ & $<0.001$ \\
\hline N-terminal pro-brain natriuretic peptide, $>486 \mathrm{pg} / \mathrm{mL}$ & $6.329(2.409-16.666)$ & $<0.001$ \\
\hline Highly sensitive C-reactive protein, $>10 \mathrm{mg} / \mathrm{L}$ & $10.11(1.362-75.072)$ & 0.024 \\
\hline Serum ferritin, $>400 \mu \mathrm{g} / \mathrm{L}$ & $7.173(0.942-54.639)$ & 0.057 \\
\hline $\mathrm{IL}-6,>62 \mathrm{U} / \mathrm{mL}$ & 0 (0-Inf) & 0.998 \\
\hline Procalcitonin, $>0.05 \mathrm{ng} / \mathrm{mL}$ & $8.268(1.105-61.874)$ & 0.04 \\
\hline $\mathrm{IL}-10,>9.1 \mathrm{pg} / \mathrm{mL}$ & $8.242(2.751-24.7)$ & $<0.001$ \\
\hline
\end{tabular}

COVID-19, coronavirus disease 2019; HR, hazard ratio; CI, confidence interval; ECOG, Eastern Cooperative Oncology Group; NRS2002, Nutritional Risk Screening 2002; adjuvant therapy, chemo-/radiotherapy plus targeted/immunotherapy. 


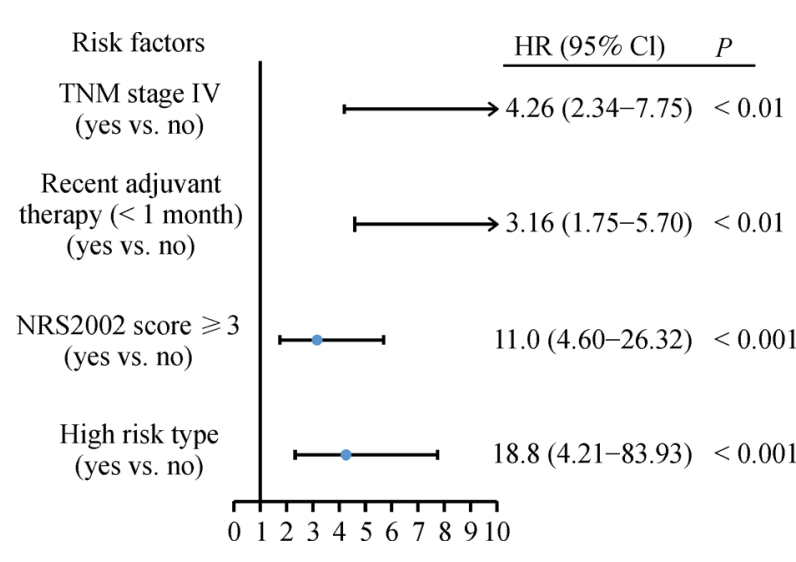

Fig. 1 Risk factors for death according to the multivariate Cox proportional hazards regression model. Shown in the figure are the hazards ratio (HR) and the $95 \%$ confidence interval $(95 \% \mathrm{CI})$ associated with the endpoint. The HRs of each variable were obtained using separate proportional hazard Cox models after adjusting for age, comorbidities, D-dimer, and lymphocyte count. Comorbidities included chronic obstructive pulmonary disease, hypertension, diabetes, and cardiovascular disease.

A TJ-COVID19-CA cohort $(n=109)$ + NRS2002 score $(<3)+$ NRS2002 score $(\geqslant 3)$

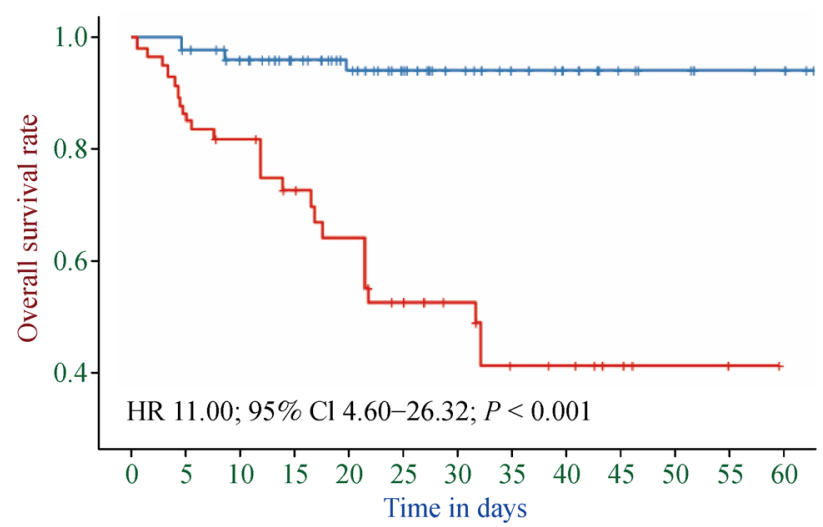

$\mathrm{C}$

$$
\begin{gathered}
\text { TJ-COVID19-CA cohort }(n=109) \\
+ \text { Tumor stage I/II/III } \quad+\text { Tumor stage IV }
\end{gathered}
$$

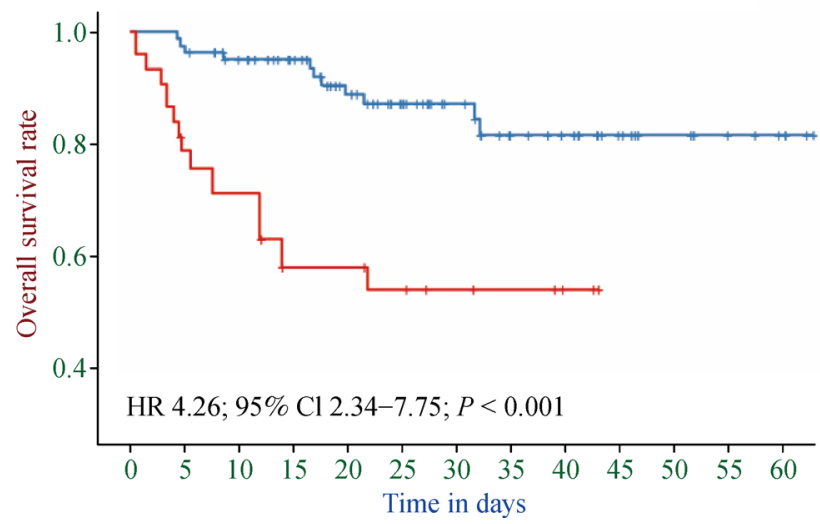

overestimation of the risk in the hazard estimates. In this model, NRS2002 score $\geqslant 3$ (adjusted HR 9.29, 95\% CI 2.63-32.80; $P<0.001$ ), high-risk type (adjusted HR 7.34, 95\% CI 1.10-48.80; $P=0.039$ ), tumor stage IV (adjusted HR 4.18, 95\% CI 1.61-10.80; $P=0.003)$, and recent adjuvant therapy ( $<1$ month) (adjusted HR 3.21, 95\% CI $1.24-83.00 ; P=0.016)$ were associated with significantly increased risk of all-cause mortality $(P<0.001$; adjusted HR 17.05; 95\% CI 11.21-25.93; $P<0.001$ for AST $>120 \mathrm{U} / \mathrm{L})($ Table S5).

\section{Discussion}

In this study, we described major differences in clinical features between cancer patients who succumbed to COVID-19 and those who recovered from it. We found that NRS2002 score $\geqslant 3$, high-risk type, tumor stage IV, and recent adjuvant therapy ( $<1$ month) were independent risk factors for in-hospital death of cancer patients using a multivariate Cox regression analysis.

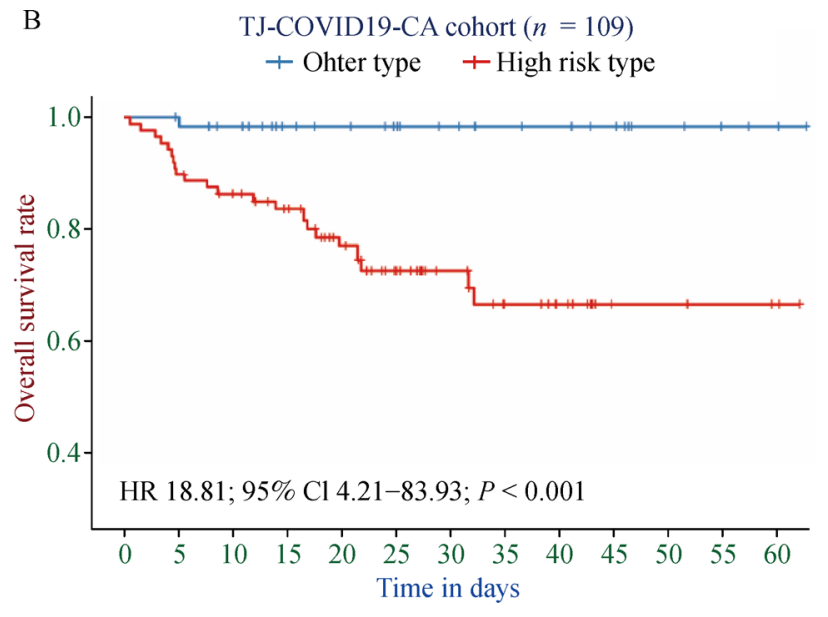

D TJ-COVID19-CA cohort $(n=109)$

+ Without recent adjuvant therapy + With recent adjuvant therapy

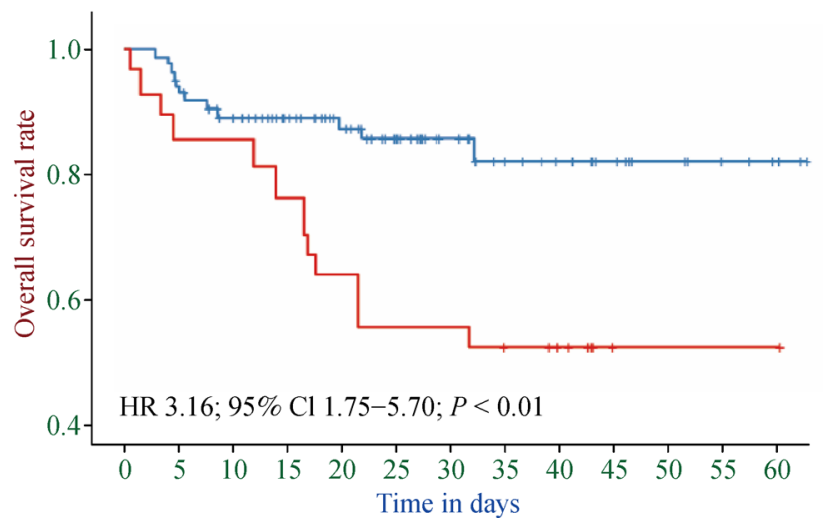

Fig. 2 Adjusted Kaplan-Meier survival curves of cancer patients with COVID-19 stratified by risk factors. (A) NRS2002 score, (B) tumor type, (C) tumor stage, and (D) recent adjuvant therapy. NRS2002, Nutritional Risk Screening 2002. 
As the epidemic continues to spread around the world, COVID-19 has become a grave threat to individuals with preexisting conditions [12]. A recent report found that 18 (1\%) of 1590 COVID-19 patients were diagnosed with cancer, which seems to be higher than the proportion in the general population in China. This observation suggests that patients with cancer have a higher risk of SARS-CoV-2 infection [6]. Consistently, our study found that 109 (3.5\%) of 3060 patients were diagnosed with cancer, which was significantly higher than the previous results and the proportion of cancer patients in the general population of China (285.83 per 100000 people $(0.29 \%)$, according to 2015 cancer epidemiology statistics [13]). In addition, the incidence of nosocomial infection among cancer patients $(17.5 \%)$ was higher than among other hospitalized patients in recent reports $(12.3 \%$ and $9.8 \%)$ [9,14]. These results indicated that cancer may be associated with an increased risk of COVID-19 infection.

Here, we report a relatively high rate of severe COVID$19(55 \%)$ and mortality $(21.1 \%)$ among cancer patients with confirmed COVID-19, which is higher than the overall mortality of our cohort $(8.2 \%)$ and the reported overall COVID-19 mortality of patients who were hospitalized in Wuhan [11,15]. Our findings support the poor prognosis of cancer patients in the current pandemic.

Among all cancer types, we observed that COVID-19 patients with hematological malignancies including leukemia, lymphoma, and multiple myeloma had the highest death rate $(41.6 \%)$. This could be explained by the fact that patients with hematological malignancies had a more impaired immune system than other cancer patients due to the malignancy itself, as well as the type of antitumor therapy used in these cases $[16,17]$. The detection rate of SARS-CoV-2 in the digestive tract and feces is high, even higher than that of pharyngeal swabs, which suggests that the digestive tract may be a target organ of this novel coronavirus $[18,19]$. Furthermore, adjuvant therapy was more frequent in patients with gastrointestinal cancer. All of these factors contributed to the high death rate among patients with malignancies of the digestive system $(33.3 \%)$. Recent studies detected the virus in a urine sample of a COVID-19 patient; thus, further investigations are needed to confirm if the urinary tract and the female reproductive tract are target organs of SARS-CoV-2 [2022]. Considering the high level of malignancy and widespread adjuvant treatment, the odds of death of patients with urinary carcinoma and gynecological malignancies were also relatively high $(35.7 \%$ and $20 \%$, respectively). The death rate of COVID-19 patients with lung cancer was $14.3 \%$, which was obviously higher than that of the general population. These results may be due to the double strike of cancer and the virus to the lungs. In this study, these tumors were classified as high-risk type, which has been confirmed to be an independent risk factor for death of cancer patients after adjusting for potential confounders, such as age, comorbidities, D-dimer, and lymphocyte count. In addition, we found that the mortality of COVID-19 patients with different types of cancer is somewhat correlated with the mortality sequence of each cancer type, but not exactly the same. This provides further evidence that the tumor type is associated with death of cancer patients with COVID-19 and also suggests that there are other factors affecting the survival of cancer patients.

The NRS2002 score is commonly used in assessing the nutritional risk of cancer patients [23]. A recent report found that NRS2002 is a useful and practical tool for screening COVID-19 patients who are at nutritional risk and in need of additional nutritional intervention. In addition, the authors identified that patients at nutritional risk had worse outcomes [24]. In this study, NRS2002 was used to analyze the risk of death in cancer patients with COVID-19. Studies have shown that patients with severe malnutrition, as is often observed in cancer patients, often have anemia and hypoproteinemia, which makes them more susceptible to respiratory tract infections and severe pneumonia [25]. We found that the nutritional status of the deceased cancer patients was relatively poor, and they had more severe anemia and hypoproteinemia than recovered patients. Accordingly, an NRS2002 score $\geqslant 3$ was identified as an independent risk factor for death in cancer patients. This finding suggested that the nutritional status of cancer patients on admission is an essential indicator, and more attention should be paid to these aspects.

Patients with stage IV tumors are in relatively poor overall condition, including physical strength, nutrition, and immunity, due to the large tumor load, which makes them more susceptible to infectious diseases, leading to a poorer prognosis [26]. A previous study found that $70 \%$ of stage IV patients developed severe events, but the authors were unable to prove a link between tumor stage and severity of COVID-19 in cancer patients due to study limitations [7]. In our study, we found that tumor stage IV was associated with a higher risk of in-hospital death even after adjusting for potential confounders.

A previous study reported that cancer patients who received recent adjuvant therapy are in an immunosuppressed state [27]. Antitumor treatment within 14 days was shown to be related to increased risk of developing severe events in COVID-19 patients [7]. In our study, we further confirmed that recent adjuvant therapy $(<1$ month) including chemo-/radiotherapy and targeted/immunotherapy is an independent risk factor for death in cancer patients with comorbid COVID-19 after adjusting for potential confounders. Studies demonstrated that a dysregulated immune response can result in excessive inflammation and $\mathrm{T}$ cell responses that can inhibit the overactivation of innate immunity $[28,29]$. This 
contradiction in cancer patients can be explained by the fact that the blunted immune status causes cancer patients to lose their ability to control the inflammatory storm caused by viral infection. The mortality of cancer patients with nosocomial SARS-CoV-2 infection (42.1\%) was higher than that of patients with community-acquired infection (16.6\%). This could be explained by the fact that all hospitalized cancer patients are receiving anticancer treatment. Contrary to a recent study [11], age ( $>65$ years old), D-dimer $(>1 \mu \mathrm{g} / \mathrm{mL})$, and lymphocyte count $\left(<1.1 \times 10^{9} / \mathrm{L}\right)$ were not independent risk factors for in-hospital death. Because cancer patients differ in many ways from the general population [30], the risk factors of death in the general population may not be appropriate for cancer patients.

Our findings underscore the urgency of giving increased attention to COVID-19-infected cancer patients. Nevertheless, our study has some limitations. First, the retrospective study design and the heterogeneity of tumor types make it difficult to eliminate bias despite adjusting for many confounders. Second, because of the logistical limitations at the onset of these emerging infections, data such as lymphocyte subgroup counts were lacking in some patients. Finally, our study can only discuss the course and prognosis of COVID-19 infection in patients with common cancer types in China, and we expect follow-up studies in other countries for cancers that are more common elsewhere, such as melanoma and pancreatic cancer.

\section{Acknowledgements}

We thank all patients involved in the study. This work was supported by the State Key Project on Infectious Diseases of China (No. 2018ZX10723204-003), the National Natural Science Foundation of China (Nos. 81874065 and 81874149), and the Hepato-BiliaryPancreatic Cancer Investigation Fund of Chen Xiao-ping Foundation for the Development of Science and Technology of Hubei Province (No. CXPJJH11800001-2018356).

\section{Compliance with ethics guidelines}

Junnan Liang, Guannan Jin, Tongtong Liu, Jingyuan Wen, Ganxun Li, Lin Chen, Wei Wang, Yuwei Wang, Wei Liao, Jia Song, Zeyang Ding, Xiao-ping Chen, and Bixiang Zhang declare no competing financial interests. The study protocol was approved by the Institutional Review Board of Tongji Hospital, Tongji Medical College, Huazhong University of Science and Technology (Wuhan, China) (Approval No.TJ-IRB20200408).

Electronic Supplementary Material Supplementary material is available in the online version of this article at https://doi.org/ $10.1007 / \mathrm{s} 11684-021-0845-6$ and is accessible for authorized users.

\section{References}

1. Zhu N, Zhang D, Wang W, Li X, Yang B, Song J, Zhao X, Huang B, Shi W, Lu R, Niu P, Zhan F, Ma X, Wang D, Xu W, Wu G, Gao GF, Tan W; China Novel Coronavirus Investigating and Research Team. A novel coronavirus from patients with pneumonia in china, 2019. N Engl J Med 2020; 382(8): 727-733

2. Dong E, Du H, Gardner L. An interactive web-based dashboard to track COVID-19 in real time. Lancet Infect Dis 2020; 20(5): 533534

3. Kamboj M, Sepkowitz KA. Nosocomial infections in patients with cancer. Lancet Oncol 2009; 10(6): 589-597

4. Li JY, Duan XF, Wang LP, Xu YJ, Huang L, Zhang TF, Liu JY, Li F, Zhang Z, Yue DL, Wang F, Zhang B, Zhang Y. Selective depletion of regulatory $T$ cell subsets by docetaxel treatment in patients with nonsmall cell lung cancer. J Immunol Res 2014; 2014 : 286170

5. Sica A, Massarotti M. Myeloid suppressor cells in cancer and autoimmunity. J Autoimmun 2017; 85: 117-125

6. Liang W, Guan W, Chen R, Wang W, Li J, Xu K, Li C, Ai Q, Lu W, Liang H, Li S, He J. Cancer patients in SARS-CoV-2 infection: a nationwide analysis in China. Lancet Oncol 2020; 21(3): 335-337

7. Zhang L, Zhu F, Xie L, Wang C, Wang J, Chen R, Jia P, Guan HQ, Peng L, Chen Y, Peng P, Zhang P, Chu Q, Shen Q, Wang Y, Xu SY, Zhao JP, Zhou M. Clinical characteristics of COVID-19-infected cancer patients: a retrospective case study in three hospitals within Wuhan, China. Ann Oncol 2020; 31(7): 894-901

8. Huang C, Wang Y, Li X, Ren L, Zhao J, Hu Y, Zhang L, Fan G, Xu J, Gu X, Cheng Z, Yu T, Xia J, Wei Y, Wu W, Xie X, Yin W, Li H, Liu M, Xiao Y, Gao H, Guo L, Xie J, Wang G, Jiang R, Gao Z, Jin Q, Wang J, Cao B. Clinical features of patients infected with 2019 novel coronavirus in Wuhan, China. Lancet 2020; 395(10223): 497506

9. Wang D, Hu B, Hu C, Zhu F, Liu X, Zhang J, Wang B, Xiang H, Cheng Z, Xiong Y, Zhao Y, Li Y, Wang X, Peng Z. Clinical characteristics of 138 hospitalized patients with 2019 novel coronavirus-infected pneumonia in Wuhan, China. JAMA 2020; 323(11): 1061-1069

10. Yang X, Yu Y, Xu J, Shu H, Xia J, Liu H, Wu Y, Zhang L, Yu Z, Fang M, Yu T, Wang Y, Pan S, Zou X, Yuan S, Shang Y. Clinical course and outcomes of critically ill patients with SARS-CoV-2 pneumonia in Wuhan, China: a single-centered, retrospective, observational study. Lancet Respir Med 2020; 8(5): 475-481

11. Zhou F, Yu T, Du R, Fan G, Liu Y, Liu Z, Xiang J, Wang Y, Song B, Gu X, Guan L, Wei Y, Li H, Wu X, Xu J, Tu S, Zhang Y, Chen H, Cao B. Clinical course and risk factors for mortality of adult inpatients with COVID-19 in Wuhan, China: a retrospective cohort study. Lancet 2020; 395(10229): 1054-1062

12. Sohrabi C, Alsafi Z, O’Neill N, Khan M, Kerwan A, Al-Jabir A, Iosifidis C, Agha R. World Health Organization declares global emergency: a review of the 2019 novel coronavirus (COVID-19). Int J Surg 2020; 76: 71-76

13. Zheng RS, Sun KX, Zhang SW, Zeng HM, Zou XN, Chen R, Gu XY, Wei WW, He J. Report of cancer epidemiology in China, 2015. Chin J Oncol (Zhonghua Zhong Liu Za Zhi) 2019; 41(1): 19-28 (in 


\section{Chinese)}

14. Cao J, Tu WJ, Cheng W, Yu L, Liu YK, Hu X, Liu Q. Clinical features and short-term outcomes of 102 patients with corona virus disease 2019 in Wuhan, China. Clin Infect Dis 2020; 71(15): 748755

15. Chen T, Wu D, Chen H, Yan W, Yang D, Chen G, Ma K, Xu D, Yu H, Wang H, Wang T, Guo W, Chen J, Ding C, Zhang X, Huang J, Han M, Li S, Luo X, Zhao J, Ning Q. Clinical characteristics of 113 deceased patients with coronavirus disease 2019: retrospective study. BMJ 2020; 368: m1091

16. Berdasco M, Esteller M. Clinical epigenetics: seizing opportunities for translation. Nat Rev Genet 2019; 20(2): 109-127

17. Röllig C, Knop S, Bornhäuser M. Multiple myeloma. Lancet 2015; 385(9983): 2197-2208

18. Xiao F, Tang M, Zheng X, Liu Y, Li X, Shan H. Evidence for gastrointestinal infection of SARS-CoV-2. Gastroenterology 2020; 158(6): 1831-1833.e3

19. Cheung KS, Hung IFN, Chan PPY, Lung KC, Tso E, Liu R, Ng YY, Chu MY, Chung TWH, Tam AR, Yip CCY, Leung KH, Fung AY, Zhang RR, Lin Y, Cheng HM, Zhang AJX, To KKW, Chan KH, Yuen KY, Leung WK. Gastrointestinal manifestations of SARSCoV-2 infection and virus load in fecal samples from a Hong Kong cohort: systematic review and meta-analysis. Gastroenterology 2020; 159(1): 81-95

20. Chen H, Guo J, Wang C, Luo F, Yu X, Zhang W, Li J, Zhao D, Xu D, Gong Q, Liao J, Yang H, Hou W, Zhang Y. Clinical characteristics and intrauterine vertical transmission potential of COVID-19 infection in nine pregnant women: a retrospective review of medical records. Lancet 2020; 395(10226): 809-815

21. Zheng S, Fan J, Yu F, Feng B, Lou B, Zou Q, Xie G, Lin S, Wang R, Yang X, Chen W, Wang Q, Zhang D, Liu Y, Gong R, Ma Z, Lu S, Xiao Y, Gu Y, Zhang J, Yao H, Xu K, Lu X, Wei G, Zhou J, Fang Q, Cai H, Qiu Y, Sheng J, Chen Y, Liang T. Viral load dynamics and disease severity in patients infected with SARS-CoV-2 in Zhejiang Province, China, January-March 2020: retrospective cohort study.
BMJ 2020; 369: m1443

22. Dong L, Tian J, He S, Zhu C, Wang J, Liu C, Yang J. Possible vertical transmission of SARS-CoV-2 from an infected mother to her newborn. JAMA 2020; 323(18): 1846-1848

23. Kondrup J, Rasmussen HH, Hamberg O, Stanga Z; Ad Hoc ESPEN Working Group. Nutritional risk screening (NRS 2002): a new method based on an analysis of controlled clinical trials. Clin Nutr 2003; 22(3): 321-336

24. Liu G, Zhang S, Mao Z, Wang W, Hu H. Clinical significance of nutritional risk screening for older adult patients with COVID-19. Eur J Clin Nutr 2020; 74(6): 876-883

25. Vigano A, Piccioni M, Trutschnigg B, Hornby L, Chaudhury P, Kilgour R. Male hypogonadism associated with advanced cancer: a systematic review. Lancet Oncol 2010; 11(7): 679-684

26. Zhao L, He R, Long H, Guo B, Jia Q, Qin D, Liu SQ, Wang Z, Xiang T, Zhang J, Tan Y, Huang J, Chen J, Wang F, Xiao M, Gao J, Yang X, Zeng H, Wang X, Hu C, Alexander PB, Symonds ALJ, Yu J, Wan Y, Li QJ, Ye L, Zhu B. Late-stage tumors induce anemia and immunosuppressive extramedullary erythroid progenitor cells. Nat Med 2018; 24(10): 1536-1544

27. Mulder WJM, Ochando J, Joosten LAB, Fayad ZA, Netea MG. Therapeutic targeting of trained immunity. Nat Rev Drug Discov 2019; 18(7): 553-566

28. Shaw AC, Goldstein DR, Montgomery RR. Age-dependent dysregulation of innate immunity. Nat Rev Immunol 2013; 13 (12): 875-887

29. Kim KD, Zhao J, Auh S, Yang X, Du P, Tang H, Fu YX. Adaptive immune cells temper initial innate responses. Nat Med 2007; 13(10): 1248-1252

30. Saner FAM, Herschtal A, Nelson BH, deFazio A, Goode EL, Ramus SJ, Pandey A, Beach JA, Fereday S, Berchuck A, Lheureux S, Pearce CL, Pharoah PD, Pike MC, Garsed DW, Bowtell DDL. Going to extremes: determinants of extraordinary response and survival in patients with cancer. Nat Rev Cancer 2019; 19(6): 339348 